https://doi.org/10.7559/gestaoedesenvolvimento.2019.381

Data de receção: 26/03/2019 Data de aceitação: 09/07/2019

\title{
A LINGUAGEM FIGURATIVA, COMPREENSÃO E EXPRESSÃO. ESTUDO COMPARATIVO: ALUNOS DE CLASSE REGULAR E ALUNOS REFERENCIADOS COM NECESSIDADES EDUCATIVAS ESPECIAIS
}

\author{
FIGURING LANGUAGE, UNDERSTANDING AND \\ EXPRESSION. COMPARATIVE STUDY: STUDENTS OF \\ REGULAR CLASS AND STUDENTS REFERENCED WITH \\ SPECIAL EDUCATIONAL NEEDS
}

Ana Paula Couceiro Figueira ${ }^{1}$ orcid.org/0000-0001-5998-3046

Tiago Ferreira ${ }^{2}$

Resumo: Linguagem é o principal instrumento de comunicação do Ser Humano, enquanto via de interação com os outros e consigo mesmo. A linguagem é vista como a janela do conhecimento humano, permitindo vivências e aprendizagens individuais e sociais.

$O$ presente estudo pretende contribuir para a depuração de um módulo, Linguagem Figurativa, do programa de intervenção neuropsicológica REHACOG.

Foi realizado um estudo descritivo e exploratório com 20 indivíduos, comparando-se alunos referenciados com Necessidades Educativas Especiais e alunos que frequentam turmas regulares, sem dificuldades de aprendizagem ou défices cognitivos denunciados.

Os resultados demonstram que as crianças com Necessidades Educativas Especiais obtêm resultados inferiores, nos $2^{\circ}$ e $3^{\circ}$ ciclos do ensino básico e ensino secundário.

Palavras-chave: Linguagem figurativa; REHACOG; Necessidades Educativas Especiais; intervenção neuropsicológica.

Abstract: Language is the main communication tool of the human being as a means of interaction with others and with himself. It is

\footnotetext{
${ }^{1}$ Faculdade de Psicologia e de Ciências da Educação da Universidade de Coimbra. Email: apcouceirofigueira@gmail.com

${ }^{2}$ Faculdade de Psicologia e de Ciências da Educação da Universidade de Coimbra.
} 
through verbal, acoustic and visual representations that the acquisition of language, becomes fundamental in establishing contact with family/caregivers, from its early years. This research contributed to the adaptation portuguese version of the program REHACOG, to the application figurative language module. A descriptive and exploratory study with 20 subjects, male and female, referenced to students with special needs and students who attend regular classes without learning difficulties or cognitive deficits. The results of this study demonstrated that children's special educational needs found below, as compared to their peers in regular classes, in different courses of studies.

Keywords: figurative language; REHACOG; learning difficulties; neuropsychological intervention.

\section{INTRODUÇÃO}

O presente estudo tem como objetivo último contribuir para a adaptação da versão portuguesa do programa REHACOG (Figueira \& Paixão, in press). Este programa assume-se como um programa de intervenção neuropsicológica. Surge da adaptação do REHACOP (Ojeda et al., 2012), originalmente, assumido como um programa de reabilitação neuropsicológica de psicoses e esquizofrenia. Neste momento, o REHACOG (Figueira \& Paixão, in press), programa multimódulos está em fase de aplicação em diferentes contextos e populações. Neste artigo, daremos conta da aplicação do módulo linguagem figurativa.

A amostra do estudo é constituída por 20 indivíduos, do género masculino e feminino, que frequentam o Instituto Educativo de Lordemão em Coimbra, alunos em classe regular e alunos referenciados com Necessidades Educativas Especiais (NEE) . Especificamente, iremos realizar uma análise comparativa, das respostas dadas pelos alunos que frequentam turmas regulares e os alunos referenciados com NEE.

\section{A LINGUAGEM INCLUINDO A LINGUAGEM FIGURATIVA}

Ao longo do desenvolvimento da criança, a linguagem faz parte integrante, permitindo a comunicação dos seus pensamentos, ideias, recordações, crenças e emoções com os seus pares. Também a educação é um dos aspetos mais importantes do desenvolvimento dos recursos 
pessoais que todas as crianças têm direito (Karande \& Kulkarni, 2005). Desta forma, a escola é o núcleo onde todas as crianças têm a oportunidade de se desenvolverem social, cognitiva e afetivamente.

Para o indivíduo, enquanto ser social, a linguagem é um processo fundamental de comunicação e de interação. Reconhecer as letras e as palavras, compreender o que se ouve e o que se lê, argumentar, verbalizar, conhecer o mundo não é suficiente. Segundo Lima (2000), para que exista linguagem não se pode prescindir da diversidade de domínios que nela estão integrados, por exemplo, motores, cognitivos, afetivos e sociais, funcionando através de células sensoriais, motoras e associativas que provêm do cérebro.

A Linguagem pode ser entendida pela sua função referencial como linguagem denotativa e conotativa. A linguagem denotativa afirma o emissor na objetividade da expressão da mensagem, referindo-se a uma realidade concreta ou imaginária. Este tipo de linguagem é sobretudo informativo. Quanto à linguagem conotativa remete-nos para o uso das palavras num sentido figurado, dependendo sempre do contexto aplicado (Chalhub, 1999). A linguagem figurativa define-se como a palavra ou o conjunto de palavras que exprime uma ideia, apelando a outros termos, recorrendo, assim, a uma semelhança, podendo ser ela real ou imaginária. Tomemos, como exemplo, o seguinte provérbio "Que vida de cão a tua!", significa que a pessoa tem más condições de vida, que vive mal. Shamisa (2004) refere que a linguagem figurativa sugere significados e ao leitor compete descobrir os seus verdadeiros significados. Segundo Gibbs (1994), os nossos conceitos básicos de experiência, na maioria figurativos, determinam a nossa maneira de pensar criativamente e de expressar as nossas ideias, tanto a nível do discurso quotidiano, como no literário.

\section{INTERVENÇÃO E REABILITAÇÃO NEUROPSICOLÓGICA}

A Neuropsicologia, desde o início do século XX, assume-se como uma disciplina emergente. Luria (1970) define Neuropsicologia como a ciência da organização cerebral dos processos mentais humanos, com o objetivo de investigar o papel dos sistemas cerebrais individuais. Outro autor, Miotto et al. (2012) refere que a Neuropsicologia é o campo da Psicologia e das Neurociências que examina as relações entre o sistema nervoso 
central, o funcionamento cognitivo e o comportamento. Cada período do desenvolvimento da história da Neuropsicologia é marcado por determinadas controvérsias a respeito do entendimento da época sobre as relações entre o cérebro e o comportamento (Kristensen, Almeida, \& Gomes, 2001). A Neuropsicologia atual estuda vários tópicos clássicos da psicologia, como a atenção, a perceção ou a memória. A Neuropsicologia recorre a métodos da psicologia experimental e do campo da psicometria, para a construção de testes. O processo de intervenção requer empenho e dedicação por parte do sujeito e vontade em capacitar as suas competências funcionais, superando as dificuldades do dia-a-dia.

A intervenção preventiva divide-se em 3 tipos: primária, secundária e terciária. A intervenção primária visa evitar a exposição de um indivíduo ou de uma população, a um fator de risco ou causal, antes que se desenvolva um mecanismo patológico. Assim, pretende evitar a ocorrência do problema-alvo. A intervenção secundária é um prolongamento da intervenção primária, diminuindo a prevalência do problema-alvo, impedindo o estabelecimento de um estado de dependência no indivíduo. Por último, a intervenção terciária objetiva a utilização de estratégias direcionadas para a reabilitação do indivíduo, potenciando a sua capacidade funcional (Almeida, 2005).

A Reabilitação Neuropsicológica, segundo Wilson (2003), é o conjunto de intervenções que têm como objetivo proporcionar ao indivíduo a melhoria dos seus processos cognitivos, emocionais e sociais com a finalidade de uma maior independência e qualidade de vida. Segundo a Organização Mundial de Saúde (2000), a Reabilitação é o processo de recuperação dos pacientes ao nível mais elevado possível nos planos físico, psicológico e social, utilizando todos os meios possíveis para reduzir o impacto das condições incapacitantes, para permitir que as pessoas alcancem um adequado nível de integração social. Existem dois conceitos fundamentais, quando se aborda a Reabilitação Neuropsicológica: a plasticidade cerebral e o princípio da transferência (Dahlin et al., 2009). Plasticidade cerebral é a capacidade do cérebro humano de se remodelar em função das experiências de cada sujeito relativamente ao meio ambiente. Ou seja, a capacidade de certas zonas cerebrais substituírem funções perdidas por lesões. Contudo, a recuperação de certas funções depende de alguns fatores, como a idade do 
indivíduo, a natureza da lesão, a quantidade de tecidos afetados ou fatores psicossociais. Por princípio da transferência entende-se a tarefa ou habilitação não treinada, que melhora em função do resultado obtido da reabilitação de outro domínio, tarefa ou habilidade (Dahlin et al., 2009).

\section{DIFICULDADES DE APRENDIZAGEM}

As dificuldades de aprendizagem (DA) têm sido objeto de estudo e investigação em vários campos como a psicologia, a educação ou a sociologia. Em meados dos anos 60, o termo dificuldades de aprendizagem (DA) começou a ser utilizado com maior frequência para descrever alunos que apresentavam insucesso escolar, mas, que não estava relacionado e nem podia ser atribuído a outros tipos de problemas de aprendizagem. Segundo Kirk (1963), as dificuldades de aprendizagem (DA) são um atraso, desordem ou imaturidade num ou mais processos da linguagem falada, da leitura, da ortografia, da caligrafia ou da aritmética, resultantes de uma possível disfunção cerebral e/ou distúrbios de comportamento. Bateman (1965) aponta que o aluno com dificuldades de aprendizagem é aquele que manifesta uma discrepância educacional significativa entre o seu potencial intelectual estimado e o seu nível atual de realização. Estas estão relacionadas com perturbações básicas nos processos de aprendizagem, e não atribuível a dificuldade intelectual e desenvolvimental, privação educacional ou cultural, perturbação emocional severa ou perda sensorial.

Serra (2007) refere que os alunos portadores de dificuldades de aprendizagem mostram-se distraídos, muito ativos, esquecidos, tagarelas, fazem inversões, omissões e confusões na leitura e na escrita, apresentam problemas no processamento e tratamento da informação sensorial, problemas emocionais, de memória, cognitivos, psicolinguísticos, psicomotores, apresentando posteriormente problemas, ao nível da leitura, da escrita e da matemática. Estas crianças apresentam dificuldades em manter a concentração e em captar informação, apresentando um desempenho bastante débil em tarefas que lhe despertam pouco interesse ou associadas a diversos fatores, que facilitam uma maior distração, como é o caso de situações que requerem atenção por longos períodos de tempo, a realização de tarefas repetitivas ou quando existem ruídos (Villar, 1998). 
Importa realçar que as crianças com NEE podem necessitar de apoio reforçado em momentos muito precisos do seu percurso escolar, tornandose indispensável que aqueles que as acompanham no seu percurso escolar (em grande parte os seus pais) reúnam as ferramentas necessárias para responderem de forma eficaz, o que poderá fazer toda a diferença num futuro próximo.

Guralnick (1997) enfatiza a importância das intervenções enquanto necessidade da família e da escola, não somente da criança, a fim de orientar e preparar esses ambientes e reduzir o impacto das dificuldades nas crianças. É necessário incutir nas crianças com dificuldades de aprendizagem o reforço de que estas têm capacidade de aprender tal como os seus pares. Encorajá-las nas suas áreas fortes para que possam ultrapassar dificuldades é a forma correta de autonomizar o processo de autoaprendizagem das crianças. Em suma, mais do que a presença ou ausência de um determinado perfil, a história de desenvolvimento, a história familiar, a história médica, a observação de comportamentos e a análise de protocolos escolares da criança assumem-se como medidas mais fiáveis para clarificar um diagnóstico de dificuldade de aprendizagem (Pereira \& Simões, 2005).

\section{MÉTODO}

\subsection{Objetivos}

O objetivo principal da presente investigação é contribuir para a adaptação da versão portuguesa do programa REHACOG (Figueira \& Paixão, in press). Como objetivo específico pretende-se analisar as capacidades ao nível da linguagem figurativa, comparando respostas entre crianças que frequentam turmas regulares e crianças referenciadas com NEE. Procedeu-se à aplicação em contexto de sala de aula, de modo individual e em simultâneo, do módulo de Linguagem Figurativa do programa de intervenção neuropsicológica REHACOG (Figueira \& Paixão, in press).

\subsection{Amostra}

A amostra do presente estudo foi recolhida com recurso a uma amostragem não probabilística por conveniência ou acidental. A amostra 
é constituída por 20 alunos, 10 do sexo masculino e 10 do sexo feminino, dos $2 .^{\circ}$ e $3 .^{\circ}$ ciclos do ensino básico e do ensino secundário, com idades compreendidas entre os 11 e os 19 anos de idade. Destes 20 alunos $(\mathrm{N}=20), 10$ alunos frequentam turmas de ensino regular, não tendo indicação, pelos professores e/ou encarregados de educação, de apresentarem quaisquer dificuldades de aprendizagem ou défice. Os restantes 10 alunos são crianças que se encontram referenciadas com NEE. São alunos que apresentam dificuldades de aprendizagem, como dislexia ou discalculia. Estes alunos foram referenciados através dos professores, diretores de turma e o psicólogo escolar. Os critérios descritos no dec. Lei 3/2008, de 7 de janeiro, e mais atualmente, o dec. Lei n ${ }^{\circ} 54 / 2018$ (ver ficha de encaminhamento em IDENTIFICAÇÃO DA NECESSIDADE DE MEDIDAS DE SUPORTE À APRENDIZAGEM E À INCLUSÃO (Art. 20o do DL 54/2018 de 6 de julho), quanto à referenciação, são claros: "A referenciação efetua-se por iniciativa dos pais ou encarregados de educação, dos serviços de intervenção precoce, dos docentes ou de outros técnicos ou serviços que intervêm com a criança ou jovem ou que tenham conhecimento da eventual existência de necessidades educativas especiais". A amostra total é composta por alunos do segundo ciclo ( $6^{\circ}$ ano de escolaridade), sendo constituído por 8 crianças dos 11 aos 13 anos de idade, em que 4 são do sexo feminino e 4 do sexo masculino; o terceiro ciclo $\left(7^{\circ}, 8^{\circ}\right.$ e $9^{\circ}$ ano de escolaridade) é constituído por 8 crianças dos 13 aos 16 anos de idade, em que 6 são do sexo feminino e 2 do sexo masculino. Por fim, no secundário $\left(11^{\circ}\right.$ ano de escolaridade) é constituído por 4 adolescentes com idades compreendidas entre os 16 e os 19 anos de idade, do sexo masculino (cf. Tabela 1).

\section{Tabela 1}

Descrição da amostra em função da condição NEE ou Regular e a escolaridade

\begin{tabular}{lccccc}
\hline & & \multicolumn{3}{c}{ Escolaridade } & \\
\cline { 2 - 6 } & & $\mathbf{2}^{\mathbf{0}}$ ciclo & $\mathbf{3}^{\mathbf{0}}$ ciclo & Secundário & Total \\
\hline \multirow{2}{*}{ Grupo } & NEE & 4 & 4 & 2 & 10 \\
\cline { 2 - 6 } & Regular & 4 & 4 & 2 & 10 \\
\hline \multirow{2}{*}{ Total } & & 8 & 8 & 4 & 20 \\
\hline
\end{tabular}




\subsection{Instrumentos}

REHACOG - Programa de intervenção/promoção cognitiva (Figueira \& Paixão, in press).

O REHACOP (2012, apresentação pública oficial, no XVI Congresso Nacional de Psiquiatria de Bilbao), originalmente, assume-se como um programa de reabilitação neuropsicológica de psicoses (Figueira \& Paixão, in press). Segundo os autores (Figueira \& Paixão, sd), o programa de intervenção resulta de um longo trabalho, de vários anos, de vários especialistas em neuropsicologia, com mais de 18 anos de experiência na área, e várias vezes premiado. Todavia, neste momento, os autores da versão original espanhola têm projetos de utilização com outras populações. O mesmo está a ser tentado em Portugal.

Trata-se de um programa integral e estruturado, constituído por 8 módulos de intervenção: atenção, linguagem, memória, funções executivas, atividades da vida diária, competências sociais, cognição social e psicoeducação. É composto por um total de 300 exercícios de intervenção, acessíveis de administração, com instruções, soluções e orientações para avaliação das mudanças, fichas de seguimento e sugestões de seguimento de tarefas para realizar em contexto familiar. Inclui um conjunto de recomendações e orientações sobre como avaliar as alterações ocorridas ou ganhos adquiridos. Os materiais são acessíveis e coloridos e incluem as instruções para os pacientes e as soluções para o profissional. Pode ser utilizado em sessões individuais ou em grupo.

O programa completo é composto por dois manuais, um para o profissional e outro para o paciente. Dos diversos módulos de intervenção que constituem o REHACOG (Figueira \& Paixão, in press), anteriormente referidos, optámos pelo módulo de Linguagem Figurativa, visto que este suscitou bastante interesse e demonstra um perfil adequado à amostra. $\mathrm{O}$ módulo de Linguagem Figurativa é composto por seis tarefas. Aplicámos as primeiras cinco tarefas: Refrões e provérbios I/II; Redundâncias; Nomes Concretos e Abstratos; Frases feitas/ditados/provérbios; Saber o que fazer e por último, Problemas com equações. Os sujeitos da amostra com NEE apresentam dificuldades de aprendizagem, como dislexia e discalculia. Deste modo, optámos por não aplicar a última tarefa, visto que o número de tarefas anteriores é elevado e o seu conteúdo, de algum modo torna-se marginal à categoria linguagem figurativa. 


\subsection{Procedimentos}

A investigação decorreu no Instituto Educativo de Lordemão Coimbra, entre os meses de março e junho de 2016, após autorização por parte da direção pedagógica do referido instituto. Segundo os princípios éticos, disponibilizámos a declaração de consentimento informado aos encarregados de educação dos alunos. Após autorização por parte dos encarregados de educação, aplicámos o protocolo, sendo este constituído pelo módulo de Linguagem Figurativa do REHACOG (Figueira \& Paixão, in press). Todo o processo de aplicação das tarefas foi realizado de forma individual e em simultâneo. Criou-se um ambiente tranquilo e sem a presença de ruídos ou elementos distratores e com iluminação apropriada. Após a recolha dos dados amostrais, estes foram inseridos numa base de dados do programa SPSS versão 22.0 e posteriormente analisados.

O REHACOG (Figueira \& Paixão, in press), especificamente o módulo de Linguagem Figurativa, é composto por um conjunto de seis pranchas/tarefas, às quais o individuo responde de forma individual no próprio enunciado. Foram aplicadas cinco dessas pranchas/tarefas. A primeira tarefa designa-se "Refrões/provérbios I e II". Neste exercício, apresenta-se uma lista de frases que aparecem incompletas. $\mathrm{O}$ indivíduo deverá completar as frases, de modo a formar provérbios ou refrões. $\mathrm{O}$ exercício é constituído por 27 itens. A segunda tarefa designa-se "Redundâncias". Este exercício apresenta um conjunto de frases incompletas, que devem ser completadas pelo sujeito. No entanto, não se pode colocar qualquer final, mas sim formar frases redundantes, ou seja, que repitam o que já está dito na palavra já existente. O exercício é constituído por 17 itens. A terceira tarefa denomina-se "Nomes concretos e abstratos". Este exercício apresenta uma lista de palavras que se devem classificar em nomes concretos ou abstratos. O exercício é constituído por 48 itens. A quarta tarefa designa-se "Frases feitas/ditados populares/provérbios". Neste exercício, o indivíduo deve escrever ao lado o significado que atribui à frase. O exercício é constituído por 44 itens. A quinta tarefa compreende "Saber o que fazer". Neste exercício, o sujeito deve responder de forma socialmente aceite a perguntas relacionadas com o "que faria se...". O exercício é constituído por 21 itens. O sexto exercício, "Problemas com equações", consiste na resolução de problema 
matemáticos, que não foi aplicado. O exercício é constituído por 3 itens. Para a cotação de cada tarefa do módulo de Linguagem Figurativa, do programa REHACOG (Figueira, \& Paixão, in press), em cada item foi contabilizado o número de respostas certas com 1 e o número de respostas erradas com 0 .

\section{RESULTADOS}

Para a caracterização do perfil da amostra, bem como para a exploração dos dados, no que diz respeito à aplicação das tarefas do módulo de linguagem figurativa, do programa de intervenção REHACOG (Figueira, \& Paixão, in press), recorremos às estatísticas descritivas, porque estas permitem-nos resumir, descrever e compreender os dados de uma distribuição, usando medidas de tendência central e medidas de dispersão. Foi ainda ensaiada uma análise inferencial entre a pontuação total obtida, pela amostra, no módulo de Linguagem Figurativa e o nível de escolaridade. Neste sentido, foi utilizado o teste de Spearman, para análise de testes não paramétricos (a amostra não apresenta uma distribuição da curva normal). Para melhor interpretação e compreensão das tabelas apresentadas, posteriormente, os valores indicam a taxa média de resposta alcançada para cada exercício/tarefa; os acertos mínimos e máximos referem-se ao número de respostas erradas e certas, alcançadas em cada exercício/tarefa.

\subsection{Análise descritiva}

De acordo com as estatísticas descritivas, na tabela 2, os resultados indicam que o grupo identificado com NEE, na generalidade $(M=60,10$; $\mathrm{dp}=30,928)$ obteve resultados inferiores comparativamente ao grupo identificado como Regular $(M=89,20 ; d p=27,038)$, no que diz respeito à pontuação total obtida no módulo de Linguagem Figurativa do programa de intervenção REHACOG (Figueira, \& Paixão, in press). Igualmente, o grupo identificado com NEE, regra geral, obtém resultados abaixo da média potencial, em todas as tarefas, com exceção da tarefa Nomes e Redundâncias, em que se aproxima ou mesmo ultrapassa as médias potenciais. De facto, das diversas tarefas que compõem o módulo de Linguagem Figurativa, os sujeitos com NEE obtiveram melhor pontuação 
na tarefa Nomes concretos e abstratos $(M=23,00 ; d p=11,255)$, tendo completado 43 respostas certas, num total de 48, que compõem o exercício. A tarefa em que este grupo apresentou resultados bem abaixo da média potencial foi Provérbios.

Quanto aos sujeitos que frequentam as turmas regulares, apresentaram melhores resultados, igualmente, na tarefa Nomes concretos e abstratos $(\mathrm{M}=28,60 ; \mathrm{dp}=11,946)$, tendo conseguido completar 48 respostas certas, num total de 48 , que compõem a tarefa. Já quanto às tarefas com menor resultados, os sujeitos com NEE obtiveram fraca pontuação na tarefa Frases feitas, ditados e provérbios $(M=5,90 ; d p=10,279)$, tendo acertado 29 respostas, das 44 que compõem o exercício. O grupo de sujeitos das turmas regulares apresentou, igualmente, menor desempenho na tarefa Frases feitas, ditados e provérbios $(M=13,40 ; \mathrm{dp}=14,385)$. A partir destes dados, podemos inferir o grau de dificuldade da tarefa Frases feitas, ditados e provérbios, possivelmente, pelo desconhecimento, por parte dos sujeitos, em reconhecer o significado das expressões apresentadas, de cariz conotativo.

\section{Tabela 2.}

Descrição dos resultados obtidos, pelo grupo com NEE e o grupo Regular, nas diferentes tarefas do módulo de Linguagem Figurativa do programa REHACOG

\begin{tabular}{|c|c|c|c|c|c|c|c|}
\hline & & $\begin{array}{c}\text { REHACOG } \\
\text { Pontuação } \\
\text { total_LF }\end{array}$ & $\begin{array}{l}\text { Refrões } \\
\text { provérbios } \\
\text { I e II }\end{array}$ & Redundâncias & $\begin{array}{c}\text { Nomes } \\
\text { concretos } \\
\text { abstratos }\end{array}$ & $\begin{array}{c}\text { Frases feitas } \\
\text { ditados } \\
\text { Provérbios }\end{array}$ & $\begin{array}{l}\text { Saber o } \\
\text { que fazer }\end{array}$ \\
\hline Número & de itens & 157 & 27 & 17 & 48 & 44 & 21 \\
\hline \multirow{4}{*}{$\begin{array}{l}\text { Grupo } \\
\text { NEE } \\
(\mathrm{N}=10)\end{array}$} & Média & 60,10 & 10,70 & 10,20 & 23,00 & 5,90 & 10,30 \\
\hline & $\begin{array}{l}\text { Desvio } \\
\text { Padrão } \\
\end{array}$ & 30,928 & 5,012 & 3,765 & 11,255 & 10,279 & 5,889 \\
\hline & $\begin{array}{l}\text { Acertos } \\
\text { mínimos }\end{array}$ & 30 & 4 & 5 & 12 & 0 & 2 \\
\hline & $\begin{array}{l}\text { Acertos } \\
\text { máximos }\end{array}$ & 113 & 19 & 16 & 43 & 29 & 21 \\
\hline \multirow{4}{*}{$\begin{array}{l}\text { Grupo } \\
\text { Regular } \\
(\mathrm{N}=10)\end{array}$} & Média & 89,20 & 15,60 & 14,50 & 28,60 & 13,40 & 17,10 \\
\hline & $\begin{array}{l}\text { Desvio } \\
\text { Padrão }\end{array}$ & 27,038 & 4,742 & 2,915 & 11,946 & 14,385 & 3,929 \\
\hline & $\begin{array}{l}\text { Acertos } \\
\text { mínimos }\end{array}$ & 59 & 9 & 7 & 14 & 0 & 10 \\
\hline & $\begin{array}{l}\text { Acertos } \\
\text { máximos }\end{array}$ & 136 & 24 & 16 & 48 & 39 & 21 \\
\hline
\end{tabular}


Somos de referir que não se obtiveram diferenças tendo em conta, quer a idade, quer o género, quer intra quer intergrupos sob análise. Contudo, regista-se que, independentemente do grupo, os resultados são ligeiramente mais positivos nos indivíduos mais velhos, aumenta um pouco à medida que aumenta a idade, correlativa de níveis de escolaridade superiores.

\section{CONCLUSÕES}

O objetivo último desta investigação é contribuir para a adaptação da versão portuguesa do programa REHACOG (Figueira \& Paixão, in press). No entanto, neste estudo foram utilizadas as tarefas do módulo Linguagem figurativa, as crianças com NEE e as crianças que frequentam turmas de ensino regular (sem défices ou limitações apontadas). De um modo geral, a leitura dos resultados alcançados remete-nos para resultados inferiores, por parte das crianças com NEE, em comparação com as crianças de turmas regulares. Durante a realização das tarefas, as crianças com NEE apresentaram, sempre, grande dificuldade na interpretação do conteúdo do módulo de Linguagem Figurativa. Estas crianças, especificamente, apresentam dificuldades de aprendizagem, tais como dislexia. Também é de referir que, durante o processo de aplicação das tarefas, os alunos referenciados com NEE mostraram-se muito distraídos, foi notória a sua saturação, acabando por não realizarem as tarefas de modo satisfatório. Como foi referido, no enquadramento concetual, Serra (2007) considera os alunos portadores de dificuldades de aprendizagem distraídos, muito ativos, esquecidos, tagarelas, fazem inversões, omissões e confusões na leitura e na escrita, apresentam problemas no processamento e tratamento da informação sensorial, problemas emocionais, de memória, cognitivos, psicolinguísticos, psicomotores, apresentando posteriormente problemas ao nível da leitura, da escrita e da matemática. Quanto aos alunos que frequentam as turmas ditas regulares, mostraram uma postura interessada e ativa nas tarefas, principalmente no $3^{\circ}$ ciclo e ensino secundário. Os resultados alcançados, por estes, foram superiores aos resultados dos seus colegas com NEE. O exercício onde melhor alcançaram resultados foi no exercício dos Nomes concretos e abstratos; já o exercício com menores resultados foi o exercício Frases feitas, ditados e provérbios. No que 
respeita à composição das tarefas, consideramos que existem certos aspetos a melhorar, por exemplo, a extensão do número de itens, que se demonstram longos para uma amostra de crianças e adolescentes. Outro aspeto a reter, para futuros estudos, prende-se com a necessidade de recorrer a amostras maiores e heterogéneas, utilizando procedimentos de amostragem sólidos. Também seria importante alargar a aplicação a outros grupos etários e contextos de formação e aprendizagem. No futuro, a aplicação de outros módulos do REHACOG (Figueira \& Paixão, in press) irá contribuir, certamente de forma positiva em processos de reabilitação e promoção cognitiva, bem como até poderá ser utilizado como estratégia de avaliação. Em suma, esperamos ter dado um contributo para a adaptação do programa de intervenção REHACOG (Figueira \& Paixão, in press), nas tarefas do módulo de Linguagem Figurativa e compreender a sua utilidade.

\section{REFERÊNCIAS}

Almeida, L. M. (2005). Da prevenção primordial à prevenção quaternária. Revista Portuguesa de Saúde Pública, 23(1), 9196.

Almeida, C., Ribeiro, C., \& Ferreira, C. (2011). Trabalho partilhado entre famílias de alunos com Dificuldade Intelectual e Desenvolvimental e os professores: A pertinência e a Atualidade. Revista Gestão e Desenvolvimento, 19, 229-247.

Bateman, B. (1965). An educational view of a diagnostic approach to learning disorders. Seattle, WA: Special Child Publications.

Chalhub, S. (1999). Funções da linguagem. São Paulo: Ática.

Dahlin, E., Nyberg, L., Sandberg, P., Backman, L., \& Stigsdotter Neely, A. (2009). Long-term effects after executive training in elderly people. Poster presented at the 9th Nordic Meeting in Neuropsychology. Gothenburg: Sweden.

Decreto-Lei n ${ }^{\circ}$ 54/2018, Educação Inclusiva.

Decreto-Lei n. ${ }^{\circ}$ 3/2008, Apoios especializados

Figueira, A. P., \& Paixão, R. (in press). REHACOG - Programa de intervenção/promoção cognitiva e emocional.

Gibbs, R. W. (1994). The poetics of mind: Figurative thought, language, and understanding. New York: Cambridge University Press. 
Guralnick, M. J. (1997). Effectiveness of early intervention for vulnerable children: A developmental perspective. American Journal on Mental Retardation, 102(4), 319-345.

Karande, S., Sankhe, P., \& Kulkarni, M. (2005). Patterns of prescription and drug dispensing. The Indian Journal of Pediatrics, 72(2), 117-121.

Kirk, S.A. (1963). Educating exceptional children. Boston: Houghton Mifflin.

Kristensen, C. H., Almeida, R. M. M., \& Gomes, W. B. (2001). Desenvolvimento histórico e fundamentos metodológicos da neuropsicologia cognitiva. Psicologia: Reflexão e Crítica, 14(2), 259274.

Lima, R. (2000). Linguagem infantil: da normalidade à patologia. Braga: APPACDM.

Luria, A.R. (1970). Fundamentos de Neuropsicologia. São Paulo: Editora Universidade de São Paulo.

Miotto, E.C., Lucia, M.C.S., \& Scaff, M. (2012). Neuropsicologia Clínica. São Paulo: Rocca.

Ojeda N., Peña J., Bengoetxea E, García A., Sánchez P., Segarra R. C, Ezcurra J., Gutiérrez Fraile M., \& Eguíluz J.I. C. (2012). REHACOP: Programa de Rehabilitación Cognitiva en Psicosis. Revista de Neurología, 54(6), 337-342.

Pereira, M., \& Simões, M. R. (2005). A avaliação da inteligência nas dificuldades de aprendizagem: investigação com a WISCIII. Psicologia, Educação e Cultura, 9(2), 307-335.

Serra, H. (2007). Avaliação e diagnóstico em dificuldades específicas de aprendizagem - Pistas para uma intervenção educativa. Lisboa: Edições ASA.

Shamisa, S. (2004). Rhetoric 2. Teerão: Payamnoor Publication.

Villar, I. (1998). Déficit de aténcion con hiperactividad: Manual para padres y educadores. Barcelona: Masson.

Wilson, B. A. (2003). Neuropsychological Rehabilitation: theory and practice. Swets \& Zeitlinger B.V., The Netherlands: Lisse. 